\title{
Multiple Dynamic Targets Encirclement Control of Multiagent Systems
}

\author{
Wenguang Zhang, Jizhen Liu, and Deliang Zeng \\ State Key Laboratory of Alternate Electrical Power System with Renewable Energy Sources, North China Electric Power University, \\ Beijing 102206, China
}

Correspondence should be addressed to Wenguang Zhang; zwgbuaa@126.com

Received 28 September 2015; Accepted 9 November 2015

Academic Editor: Peng Lin

Copyright (c) 2015 Wenguang Zhang et al. This is an open access article distributed under the Creative Commons Attribution License, which permits unrestricted use, distribution, and reproduction in any medium, provided the original work is properly cited.

\begin{abstract}
This paper develops the distributed encirclement control problem of multiagent systems, in which each agent tracks multiple targets, each target can be tracked by one agent, and the numbers of the agents and the targets are the same or not. Firstly, an encirclement control protocol is proposed for multiagent systems, and this protocol contains some estimators. Secondly, some conditions are derived, under which multiagent systems can achieve encirclement control by circular formation. Finally, numerical simulations are provided to illustrate the obtained results.
\end{abstract}

\section{Introduction}

Distributed coordination control of multiagent systems has attracted a great number of researchers from different backgrounds, such as physics, biology, control theory, robotics, and computer [1-17]. Multiagent systems arise in wide areas, including movement of flocks of birds or schools of fish, molecular conformation problems, cooperative control of unmanned aerial vehicles, formation control of mobile robots, and power systems. For instance, Olfati-Saber and Murray [2] presented two consensus protocols to solve agreement problems in a network of continuous-time and discrete-time integrator agents and investigated a systematical framework of consensus problem in networks of agents with a simple scalar continuous-time integrator in three cases. Lin and Jia [3-5] studied consensus problems for first-order or second-order multiagent systems with time varying communication delays and switching topology. In [6, 7], $H_{\infty}$ consensus problems were, respectively, investigated for the first-order and high-order multiagent systems, and they gave the conditions of satisfying $H_{\infty}$ based on linear matrix inequality. In [8], the constrained consensus problem of multiagent systems in dynamically changing unbalanced networks with communication delays has been studied. It has been shown that the error auxiliary vanishes as time evolves and the linear main body has an exponential convergence rate to a vector as a separate system.

In some situations, encirclement control for multiple targets can be studied in a distributed manner. However, the work on this problem is rare currently. In $[9,10]$, they only considered the fixed targets. In [9], a group of unmanned aerial vehicles surrounding one target by using decentralized nonlinear model predictive control was studied. In [10], Chen et al. used the leader-follower framework to make the followers surround the stationary leaders with a fixed communication graph. A multiagent cooperative control problem in which agents move collectively to surround multiple targets was studied in [11], and the proposed control law works not only for stationary targets but also for dynamic ones. But in that paper, it is assumed that the numbers of the agents and the targets are the same.

This paper will focus on the study of the distributed encirclement control and tracking problems of multiple dynamic targets by graph theory. We suppose that each agent tracks multiple targets and each target only can be tracked by one agent. Firstly, we design a control protocol including some estimators. Secondly, the required conditions to realize 
encirclement are proposed by Lyapunov theory. Finally, we prove this theory to be effective by the simulation.

The rest of this paper is organized as follows. In Section 2, we introduce some basic notations and some concepts in graph theory. In Section 3, the model to be researched is formulated and a distributed encirclement control protocol is proposed. In Section 4, the main results are stated and derived. In Section 5, numerical simulations are provided to demonstrate the effectiveness of the obtained theoretical results. In Section 6, we conclude this paper.

\section{Notations and Preliminaries}

Let $G(\mathscr{V}, \mathscr{E}, \mathscr{A})$ be an undirected graph, where $\mathscr{V}=$ $\left\{s_{1}, s_{2}, \ldots, s_{n}\right\}$ is the set of nodes and $\mathscr{E} \in \mathscr{V} \times \mathscr{V}$ is the set of edges. The node indexes belong to a finite index set $\mathscr{I}=\{1,2, \ldots, n\}$ and $N_{i}=\left\{s_{j} \in \mathscr{V}\left(s_{i}, s_{j}\right) \in \mathscr{E}\right\}$ is defined as the neighbourhood set of $s_{i} . \mathscr{A}=\left[a_{i j}\right] \in \mathbb{R}^{n \times n}$ is a symmetric weighted adjacency matrix, where the element $a_{i j}$ represents the weight from node $s_{i}$ to node $s_{j}$. When $s_{j} \in N_{i}$, then $a_{i j}>0$, or else $a_{i j}=0$. In the undirected graph, any $\left(s_{i}, s_{j}\right) \in$ $\mathscr{E} \Leftrightarrow\left(s_{j}, s_{i}\right) \in \mathscr{E}$. The graph Laplacian with the diagraph is defined as $L=\left[l_{i j}\right]$, where $l_{i i}=\sum_{j=1}^{n} a_{i j}$ and $l_{i j}=-a_{i j}, i \neq j$. If there is a path from every node to every other node, the graph is said to be connected and undirected.

Lemma 1 (see [18]). If the undirected graph $G$ is connected, then its Laplacian L satisfies the following:

(1) Zero is a simple eigenvalue of $L$, and $\mathbf{1}_{n}$ is the corresponding eigenvector, and $L \mathbf{1}_{n}=0$.

(2) The remaining $n-1$ eigenvalues of $L$ all have positive real parts. And $L$ is a symmetric matrix and the eigenvalues $0=\lambda_{\min }=\lambda_{1} \leq \lambda_{2} \cdots \leq \lambda_{n}=\lambda_{\max }$.

Lemma 2 (see [19]). Suppose there is a positive definite Lyapunov function $V(x, t)$ defined on $U \times R^{+}$, where $U \in$ $U_{0}$ is the neighbourhood of the origin. There are positive real constants $c>0$ and $0<\alpha<1$, such that $\dot{V}(x, t)+c V^{\alpha}(x, t)$ is negative semidefinite on $U$. Then, $V(x, t)$ is locally finite-time convergent with a settling time

$$
T \leq \frac{V^{1-\alpha}\left(x_{0}(t)\right)}{c(1-\alpha)} .
$$

\section{Model and Problem Description}

The multiagent systems under consideration comprise $n$ agents and $m$ targets. Each agent is regarded as a node in an undirected graph $G$. Each edge $\left(s_{j}, s_{i}\right)$ corresponds to an available information path from agent $j$ to $i$. Moreover, each agent updates its current state based on the information received from its neighbors. We suppose that the dynamic of the $i$ th agent is

$$
\dot{y}_{i}(t)=u_{i}(t), \quad i \in \mathscr{I}=\{1,2, \ldots, n\},
$$

where $y_{i}(t) \in R^{2}$ denotes the position and $u_{i}(t) \in R^{2}$ is the control input of $i$ th agent at time $t$.
To simplify the analysis, we will consider the dynamics in polar coordinate system corresponding to system (2):

$$
\begin{gathered}
\dot{l}_{i}(t)=v_{i}(t), \\
\dot{\theta}_{i}(t)=\omega_{i}(t),
\end{gathered}
$$

$$
i \in \mathscr{I} \text {, }
$$

where $l_{i}(t) \in R$ and $\theta_{i}(t) \in R$, respectively, denote the radius and angle of the $i$ th agent in the polar coordinate system which regards the geometric center $P=(1 / m) \sum_{i=1}^{m} r_{i}(t)$ as the origin. $r_{i}(t)$ represents the position of the $i$ th target at time $t$. Obviously, $y_{i}(t)=p_{i}(t)+\left[l_{i}(t) \cos \left(\theta_{i}(t)\right), l_{i}(t) \sin \left(\theta_{i}(t)\right)\right]^{T}$, where $p_{i}(t)$ denotes the estimated value of the distance from the $i$ th agent to the geometric center $P$.

We say the control protocol $u_{i}(t)$ can solve the distributed encirclement problems of system (2) if the states of agents satisfy

$$
\begin{aligned}
& \lim _{t \rightarrow \infty}\left\{\left\|y_{i}(t)-\frac{1}{m} \sum_{k=1}^{m} r_{k}(t)\right\|\right. \\
& \left.-k \max _{i \in \mathcal{I}}\left\{\left\|r_{i}(t)-\frac{1}{m} \sum_{k=1}^{m} r_{k}(t)\right\|\right\}\right\}=0, \\
& \lim _{t \rightarrow \infty}\left[\theta_{i}(t)-\theta_{j}(t)-\frac{2 \pi(i-j)}{n}\right]=0,
\end{aligned}
$$

$$
i \in \mathscr{I}
$$

where $k>1$.

Assumption 3. The $i$ th agent can track $n_{i} \geq 1$ targets, and each target can only be tracked by one agent.

Let $\bar{r}_{i}(t)=\left(1 / n_{i}\right) \sum_{k=1}^{n_{i}} r_{i_{k}}(t)$ and $\Psi_{i}(t)=(n / m) n_{i} \bar{r}_{i}(t)$; then $(1 / n) \sum_{i=1}^{n} \Psi_{i}(t)=(1 / m) \sum_{i=1}^{m} r_{i}(t)$. To solve the distributed encirclement control problem of system (3), we can first estimate the distributed center of the targets and then drive the agents encirclement and track the targets. The following control protocol is

$$
\begin{aligned}
& v_{i}(t)=-k_{1} \operatorname{sign}\left(l_{i}(t)-k \rho_{i}(t)\right), \\
& \omega_{i}(t)=\sum_{j \in N_{i}(t)} a_{i j}\left(\theta_{j}(t)-\theta_{i}(t)-\frac{2 \pi(j-i)}{n}\right),
\end{aligned}
$$

\section{$i \in \mathscr{I}$,}

where $\rho_{i}(t)$ denotes the estimated value of maximum distance from $\bar{r}_{i}(t)$ to the distributed center for the $i$ th agent and $k_{1}>0$ represents the control parameter.

The estimator of the $i$ th agent corresponding to the distributed center positions $\rho_{i}(t)$ is given as follows:

$$
\begin{aligned}
& \dot{\varphi}_{i}(t)=\alpha \sum_{j \in N_{i}(t)} \operatorname{sign}\left(p_{j}(t)-p_{i}(t)\right), \\
& p_{i}(t)=\varphi_{i}(t)+\Psi_{i}(t),
\end{aligned}
$$

$$
i \in \mathscr{I}
$$


where $\varphi_{i}(t)$ is the intermediate variable with $\varphi_{i}(0)=0$ and $\alpha>0$ is the control parameter.

Assumption 4. The speed of all targets has the common upper bound as the definition in [17]; then there exists $\beta>0$ such that $\left\|\dot{r}_{i}(t)\right\| \leq \beta, i \in\{1,2, \ldots, m\}$.

Noting that $d_{i}(t)=\left\|\bar{r}_{i}(t)-p_{i}(t)\right\|, i \in \mathscr{I}$, the estimator of the $i$ th agent corresponding to $\rho_{i}(t)$ is rewritten as follows:

$$
\dot{\rho}_{i}(t)=-k_{2} \operatorname{sign}\left[\rho_{i}(t)-\max _{j \in \mathscr{F}}\left\{d_{j}(t)\right\}\right], \quad i \in \mathscr{F},
$$

where $k_{2}>0$ represents the control parameter.

To simplify analysis, we transform the original system (3)-(5) into an equivalent system. Let $\widehat{\theta}_{i}(t)=\theta_{i}(t)-2 i \pi / n$; then the closed-loop system (3)-(5) is given as follows:

$$
\begin{aligned}
\dot{l}_{i}(t) & =-k_{1} \operatorname{sign}\left(l_{i}(t)-k \rho_{i}(t)\right), \\
\dot{\hat{\theta}}_{i}(t) & =\sum_{j \in N_{i}(t)} a_{i j}\left(\widehat{\theta}_{j}(t)-\widehat{\theta}_{i}(t)\right),
\end{aligned}
$$

$i \in \mathscr{I}$.

Let $\widehat{\theta}=\left[\widehat{\theta}_{1}(t), \widehat{\theta}_{2}(t), \ldots, \widehat{\theta}_{n}(t)\right]$; then $\dot{\hat{\theta}}(t)=-L \widehat{\theta}$. So the control protocol corresponding to system (2) is

$$
\begin{aligned}
u_{i}(t)= & {\left[\begin{array}{l}
v_{i}(t) \cos \left(\theta_{i}(t)\right)-l_{i}(t) \omega_{i}(t) \sin \left(\theta_{i}(t)\right) \\
v_{i}(t) \sin \left(\theta_{i}(t)\right)+l_{i}(t) \omega_{i}(t) \cos \left(\theta_{i}(t)\right)
\end{array}\right] } \\
& +\dot{p}_{i}(t), \quad i \in \mathscr{I} .
\end{aligned}
$$

\section{Main Results}

Lemma 5. Considering estimator (6), if $\alpha>n^{2} \beta$ and Assumptions 3 and 4 are both satisfied, there must exist $T_{1}>0$ such that $\lim _{t \rightarrow T_{1}}\left[p_{i}(t)-(1 / m) \sum_{k=1}^{m} r_{k}(t)\right]=0$ for any $i \in \mathscr{I}$. That is to say, the estimate $p_{i}(t)$ corresponding to distributed center for all agents will converge to the distributed center of targets in finite time.

Proof. Let $p(t)=\left[p_{1}^{T}(t), p_{2}^{T}(t), \ldots, p_{n}^{T}(t)\right]^{T}$. We define the Lyapunov function as follows:

$$
\begin{aligned}
& V_{1}(t) \\
& =\frac{1}{2} \sum_{i=1}^{n}\left[p_{i}(t)-\frac{1}{n} \sum_{k=1}^{n} p_{k}(t)\right]^{T}\left[p_{i}(t)-\frac{1}{n} \sum_{k=1}^{n} p_{k}(t)\right] ;
\end{aligned}
$$

then the derivative of $V_{1}(t)$ along the trajectories of system (6) is given by

$$
\begin{aligned}
\dot{V}_{1}(t) & =\sum_{i=1}^{n}\left\{[ p _ { i } ( t ) - \frac { 1 } { n } \sum _ { k = 1 } ^ { n } p _ { k } ( t ) ] ^ { T } \left[\dot{p}_{i}(t)\right.\right. \\
- & \left.\left.\frac{1}{n} \sum_{k=1}^{n} \dot{p}_{k}(t)\right]\right\}=\sum_{i=1}^{n}\left\{\left[p_{i}(t)-\frac{1}{n} \sum_{k=1}^{n} p_{k}(t)\right]^{T}\right. \\
. & {\left[\alpha \sum_{j \in N_{i}(t)} \operatorname{sign}\left(p_{j}(t)-p_{i}(t)\right)+\dot{\Psi}_{i}(t)\right.} \\
& \left.\left.-\frac{1}{n} \sum_{k=1}^{n} \dot{p}_{k}(t)\right]\right\} .
\end{aligned}
$$

For

$$
\begin{aligned}
& \alpha \sum_{i=1}^{n}\left\{\left[p_{i}(t)-\frac{1}{n} \sum_{k=1}^{n} p_{k}(t)\right]^{T}\right. \\
& \left.\cdot \sum_{j \in N_{i}(t)} \operatorname{sign}\left(p_{j}(t)-p_{i}(t)\right)\right\}=\frac{\alpha}{2} \\
& \cdot \sum_{i=1}^{n} \sum_{j \in N_{i}(t)}\left\{\left[p_{i}(t)-p_{j}(t)\right]^{T} \operatorname{sign}\left(p_{j}(t)-p_{i}(t)\right)\right\} \\
& \leq-\frac{\alpha}{2} \sum_{i=1}^{n} \sum_{j \in N_{i}(t)}\left\|p_{i}(t)-p_{j}(t)\right\|, \\
& \left\|\dot{\Psi}_{i}(t)\right\|=\left\|\frac{n}{m} n_{i} \dot{\bar{r}}_{i}(t)\right\|=\left\|\frac{n}{m} \sum_{k=1}^{n_{i}} \dot{r}_{i_{k}}(t)\right\| \leq \frac{n}{m} n_{i} \beta \leq n \beta,
\end{aligned}
$$

we have

$$
\begin{aligned}
& \sum_{i=1}^{n}\left\{\left[p_{i}(t)-\frac{1}{n} \sum_{k=1}^{n} p_{k}(t)\right]^{T} \dot{\Psi}_{i}(t)\right\} \\
& \quad \leq n \beta \sum_{i=1}^{n}\left\|p_{i}(t)-\frac{1}{n} \sum_{k=1}^{n} p_{k}(t)\right\| \\
& \quad \leq n \beta \sum_{j=1, j \neq i}^{n} \max _{i=1,2, \ldots, n}\left\{\left\|p_{i}(t)-p_{j}(t)\right\|\right\} \\
& =n^{2} \beta \max _{i, j=1,2, \ldots, n}\left\{\left\|p_{i}(t)-p_{j}(t)\right\|\right\} \\
& \leq \frac{n^{2} \beta}{2} \sum_{i=1}^{n} \sum_{j \in N_{i}(t)}\left\|p_{i}(t)-p_{j}(t)\right\| .
\end{aligned}
$$


For

$$
\begin{gathered}
\sum_{i=1}^{n}\left\{\left[p_{i}(t)-\frac{1}{n} \sum_{k=1}^{n} p_{k}(t)\right]^{T}\left[-\frac{1}{n} \sum_{k=1}^{n} \dot{p}_{k}(t)\right]\right\} \\
=\left[\sum_{i=1}^{n} p_{i}(t)-\sum_{k=1}^{n} p_{k}(t)\right]\left[-\frac{1}{n} \sum_{k=1}^{n} \dot{p}_{k}(t)\right]=0,
\end{gathered}
$$

we get

$$
\begin{aligned}
\dot{V}_{1}(t) \leq & -\frac{\alpha}{2} \sum_{i=1}^{n} \sum_{j \in N_{i}(t)}\left\|p_{i}(t)-p_{j}(t)\right\| \\
& +\frac{n^{2} \beta}{2} \sum_{i=1}^{n} \sum_{j \in N_{i}(t)}\left\|p_{i}(t)-p_{j}(t)\right\| \\
= & \left(\frac{n^{2} \beta}{2}-\frac{\alpha}{2}\right) \sum_{i=1}^{n} \sum_{j \in N_{i}(t)}\left\|p_{i}(t)-p_{j}(t)\right\| .
\end{aligned}
$$

Let $m(t)=\max _{i, j \in \mathscr{I}}\left\{\left\|p_{i}(t)-p_{j}(t)\right\|\right\}$. For $\| p_{i}(t)-$ $(1 / n) \sum_{k=1}^{n} p_{k}(t)\left\|\leq(1 / n) \sum_{k=1}^{n}\right\| p_{i}(t)-p_{k}(t) \| \leq m(t)$, we obtain $\dot{V}_{1}(t) \leq(n / 2) m(t)^{2}$. Furthermore, for $\sum_{i=1}^{n} \sum_{j \in N_{i}(t)} \| p_{i}(t)-$ $p_{j}(t) \| \geq m(t)$, we have

$$
\begin{aligned}
& \dot{V}_{1}(t)+\left(\frac{\alpha}{2}-\frac{n^{2} \beta}{2}\right) \sqrt{\frac{n}{2}} V_{1}(t)^{1 / 2} \\
& \quad \leq\left(\frac{n^{2} \beta}{2}-\frac{\alpha}{2}\right) m(t)+\sqrt{\frac{n}{2}}\left(\frac{\alpha}{2}-\frac{n^{2} \beta}{2}\right) \sqrt{\frac{n}{2}} m(t) \\
& \quad=0
\end{aligned}
$$

Therefore, from Lemma 2, there exists $T_{1}>0$ such that $\lim _{t \rightarrow T_{1}}\left[p_{i}(t)-(1 / m) \sum_{k=1}^{m} r_{k}(t)\right]=0$, and we get $\lim _{t \rightarrow T_{1}}\left[p_{i}(t)-p_{j}(t)\right]=0$.

Lemma 6. Considering estimator (7), one supposes that Assumptions 3 and 4 are both satisfied. If $k_{2}>2 \beta$, estimator (7) must be steady in finite time.

Proof. We define the Lyapunov function as follows:

$$
V_{2}(t)=\sum_{i=1}^{n} \varepsilon_{i} \operatorname{sign}\left(\varepsilon_{i}\right),
$$

where $\varepsilon_{i}=\rho_{i}(t)-\max _{j \in \mathscr{I}}\left\{d_{j}(t)\right\}$. Then, the derivative of $V_{2}(t)$ along the trajectories of system (7) is given by

$$
\begin{aligned}
& \dot{V}_{2}(t)=\sum_{i=1}^{n} \operatorname{sign}\left(\varepsilon_{i}\right) \dot{\varepsilon}_{i}=\sum_{i=1}^{n} \operatorname{sign}\left(\varepsilon_{i}\right) \\
& \cdot {\left[-k_{2} \operatorname{sign}\left(\rho_{i}(t)-\max _{j \in \mathscr{I}}\left\{d_{j}(t)\right\}\right)\right.} \\
&\left.-\max _{j \in \mathscr{I}}\left\{\dot{d}_{j}(t)\right\}\right] .
\end{aligned}
$$

Let $d(t)=\max _{j \in \mathscr{F}}\left\{d_{j}(t)\right\}=\max _{j \in \mathscr{F}}\left\{\left\|\bar{r}_{j}(t)-p_{j}(t)\right\|\right\}$. According to Lemma 5, there exists $T_{1}>0$ such that $p_{i}(t)=$ $(1 / m) \sum_{k=1}^{m} r_{k}(t)$ when $t>T_{1}$.

Then, we have

$$
\begin{aligned}
|\dot{d}(t)| & =\left|\max _{j \in \mathscr{I}}\left\{\dot{d}_{j}(t)\right\}\right| \\
& =\left|\max _{j \in \mathscr{I}}\left\{\left\|\frac{1}{n_{j}} \sum_{k=1}^{n_{j}} \dot{r}_{j_{k}}(t)-\frac{1}{m} \sum_{k=1}^{m} \dot{r}_{k}(t)\right\|\right\}\right| \\
& \leq \max _{j \in \mathscr{I}}\left\{\frac{1}{n_{j}} \sum_{k=1}^{n_{j}}\left\|\dot{r}_{j_{k}}(t)\right\|+\frac{1}{m} \sum_{k=1}^{m}\left\|\dot{r}_{k}(t)\right\|\right\} \leq 2 \beta .
\end{aligned}
$$

Hence,

$$
\begin{aligned}
\dot{V}_{2}(t) & \leq \sum_{i=1}^{n}\left\{\operatorname{sign}\left(\varepsilon_{i}\right)\left[-k_{2} \operatorname{sign}\left(\varepsilon_{i}\right)\right]+|\dot{d}(t)|\right\} \\
& \leq \sum_{i=1}^{n}\left\{\operatorname{sign}\left(\varepsilon_{i}\right)\left[-k_{2} \operatorname{sign}\left(\varepsilon_{i}\right)\right]+2 \beta\right\} \\
& =\sum_{i=1}^{n}\left\{-k_{2}\left|\operatorname{sign}\left(\varepsilon_{i}\right)\right|+2 \beta\right\} .
\end{aligned}
$$

According to the condition $k_{2}>2 \beta$, we conclude that system (7) must be steady in finite time.

Lemma 7. Considering the first equation of system (8), one supposes that Assumptions 3 and 4 are both satisfied. If $k_{2}>2 \beta$ and $k_{1}>2 k \beta$, this system can be steady in finite time.

Proof. It is easy to get the conclusion according to Lemma 4 in [1], so we ignore the proof here.

Theorem 8. Considering system (2), if the network topology of multiagent systems is connected and Assumptions 3 and 4 are both satisfied, letting $\alpha>n^{2} \beta, k_{1}>2 k \beta$, and $k_{2}>2 \beta$, then protocol (9) can solve the distributed encirclement control problem of system (2).

Proof. From Lemma 5, there exists $T_{1}>0$ such that $\lim _{t \rightarrow T_{1}}\left[p_{i}(t)-(1 / m) \sum_{k=1}^{m} r_{k}(t)\right]=0$. From Lemma 7, there exists $T_{2}>T_{1}$ such that $\lim _{t \rightarrow T_{2}}\left\{\left\|y_{i}(t)-(1 / m) \sum_{k=1}^{m} r_{k}(t)\right\|-\right.$ $\left.k \max _{j \in \mathscr{F}}\left\{\left\|r_{j}(t)-(1 / m) \sum_{k=1}^{m} r_{k}(t)\right\|\right\}\right\}=0$. As graph $G$ is connected, the Laplacian $L$ of $G$ has nonnegative real part. For $\dot{\hat{\theta}}(t)=-L \hat{\theta}(t)$, then we have $\lim _{t \rightarrow \infty}\left[\widehat{\theta}_{i}(t)-\widehat{\theta}_{j}(t)\right]=0$, so $\lim _{t \rightarrow \infty}\left[\theta_{i}(t)-\theta_{j}(t)-2 \pi(i-j) / n\right]=0$. 


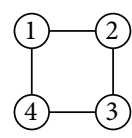

FIGURE 1: The fixed network topology of multiagent systems.

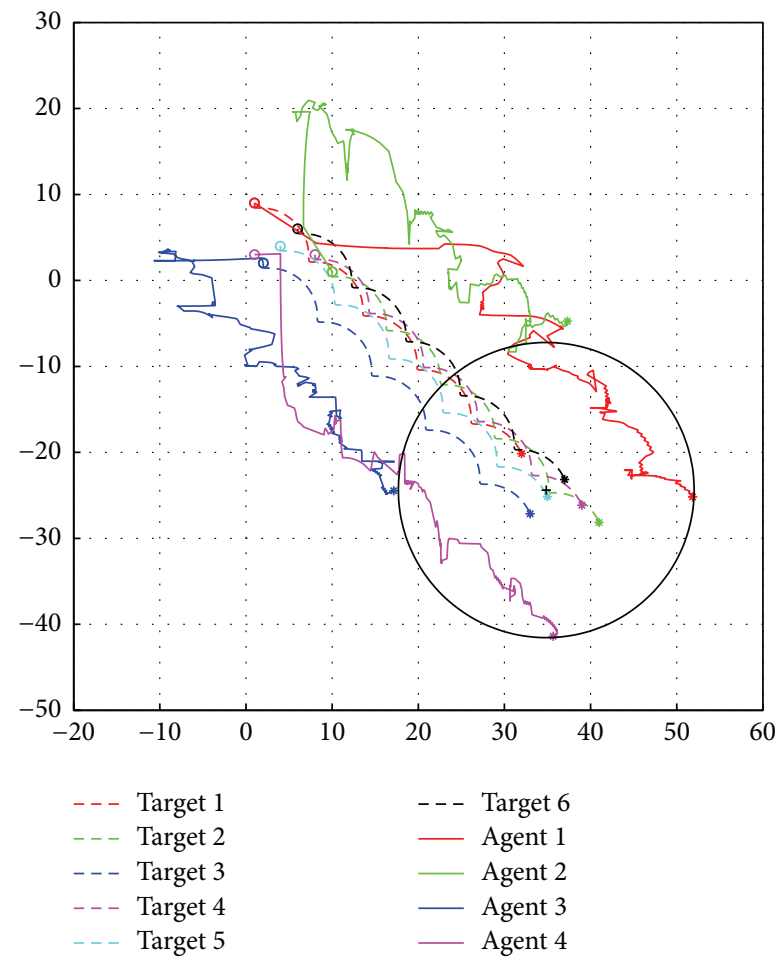

Figure 2: The trajectories of the agents and targets of multiagent systems with fixed topology.

\section{Simulation Results and Analysis}

In this section, the results of simulation by Matlab prove the effectiveness of the theoretical results obtained. The dynamic targets are

$$
\begin{aligned}
& r_{1}(t)=\left[\begin{array}{c}
t+1-\sin t \\
-t+10-\cos t
\end{array}\right], \\
& r_{2}(t)=\left[\begin{array}{c}
t+10-\sin t \\
-t+2-\cos t
\end{array}\right], \\
& r_{3}(t)=\left[\begin{array}{c}
t+2-\sin t \\
-t+3-\cos t
\end{array}\right], \\
& r_{4}(t)=\left[\begin{array}{c}
t+8-\sin t \\
-t+4-\cos t
\end{array}\right], \\
& r_{5}(t)=\left[\begin{array}{c}
t+4-\sin t \\
-t+5-\cos t
\end{array}\right], \\
& r_{6}(t)=\left[\begin{array}{c}
t+6-\sin t \\
-t+7-\cos t
\end{array}\right] .
\end{aligned}
$$

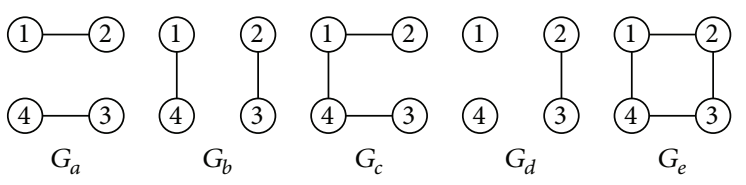

Figure 3: The five kinds of topologies of multiagent systems.

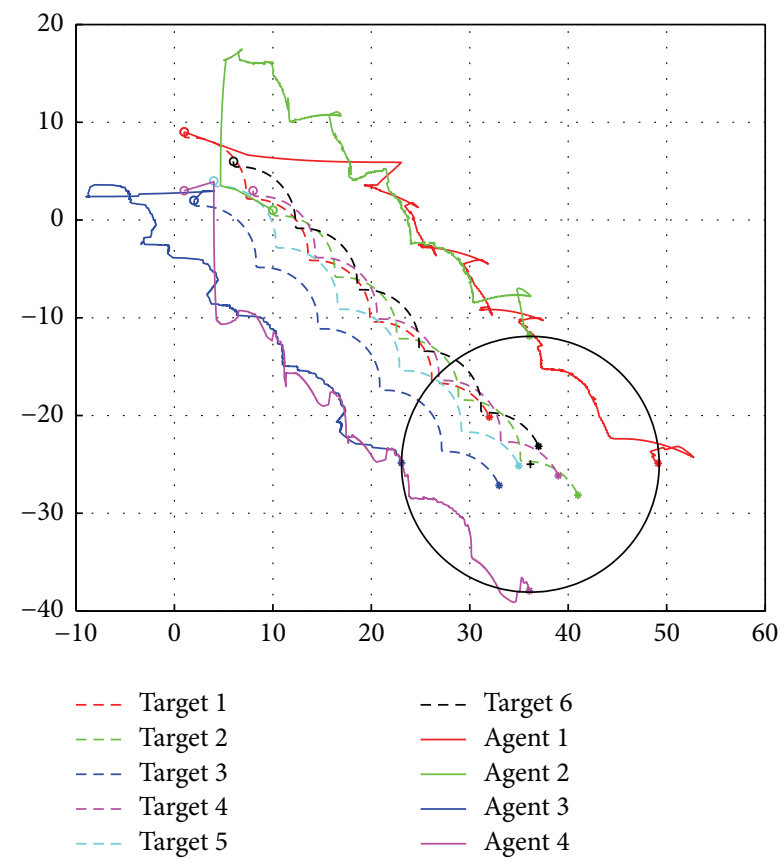

FIGURE 4: The trajectory of the agents and targets of multiagent systems with switching topology randomly.

The corresponding fixed network topology of multiagent systems with 4 nodes is shown in Figure 1 . Let $k=2, \beta=22$, $\alpha=33, k_{1}=10$, and $k_{2}=10$, and the initial conditions are $(9,9),(10,9),(-6,2)$, and $(1,-5)$. Figure 2 represents the trajectories of the agents and targets of multiagent systems and it shows that the multiagent systems with fixed topology can encircle the multiple targets in the form of circular formation.

Figure 3 shows the five kinds of topologies of multiagent systems with 4 nodes and these topologies can realize the random switch obeyed uniform distribution among them. Let $k=2, \beta=22, \alpha=33, k_{1}=10$, and $k_{2}=10$, and the initial conditions are $(1,9),(10,1),(2,2)$, and $(1,3)$. Figure 4 represents the trajectories of the agents and targets of multiagent systems and it shows that the multiagent systems with switching topology randomly can encircle the multiple targets in the circular formation.

\section{Conclusion}

In this paper, we investigate the distributed encirclement of multiagent systems with multiple dynamic targets with the assumption that each agent can track multiple targets, each target only can be tracked by one agent, and the numbers of the agents and the targets are the same or not. 
The encirclement and tracking method in circular formation is proposed. Considering that each agent can only get partial information of targets, the target state estimators which can estimate the average position of targets are designed. In finite time, every agent's motion radius is locally converged to circular formation radius of system within a settling time. All agents can maintain the formation which can be updated in real time according to the change of targets' state. By Lyapunov function, it is proved that every agent can get the whole information of targets in finite time, and meanwhile it realizes the circular formation in finite time. The simulation results illustrate that this proposed method is effective for not only multiple static targets but also multiple dynamic targets.

\section{Conflict of Interests}

The authors declare that there is no conflict of interests regarding the publication of this paper.

\section{Acknowledgments}

This work was supported by the National Basic Research Program of China (973 Program) (Grant no. 2012CB215203) and the National Natural Science Foundation of China (Grants nos. 61304155, 61203080, and 61573082).

\section{References}

[1] W. Ren and Y. Cao, Distributed Coordination of Multi-agent Networks, Springer, New York, NY, USA, 2011.

[2] R. Olfati-Saber and R. M. Murray, "Consensus problems in networks of agents with switching topology and time-delays," IEEE Transactions on Automatic Control, vol. 49, no. 9, pp. 15201533, 2004.

[3] P. Lin and Y. Jia, "Average consensus in networks of multi-agents with both switching topology and coupling time-delay," Physica A, vol. 387, no. 1, pp. 303-313, 2008.

[4] P. Lin and Y. Jia, "Further results on decentralised coordination in networks of agents with second-order dynamics," IET Control Theory \& Applications, vol. 3, no. 7, pp. 957-970, 2009.

[5] P. Lin and Y. Jia, "Consensus of a class of second-order multiagent systems with time-delay and jointly-connected topologies," IEEE Transactions on Automatic Control, vol. 55, no. 3, pp. 778-784, 2010.

[6] P. Lin, Y. Jia, and L. Li, "Distributed robust $H_{\infty}$ consensus control in directed networks of agents with time-delay," Systems \& Control Letters, vol. 57, no. 8, pp. 643-653, 2008.

[7] L. Mo and Y. Jia, " $H_{\infty}$ consensus control of a class of high-order multi-agent systems," IET Control Theory \& Applications, vol. 5, no. 1, pp. 247-253, 2011.

[8] P. Lin and W. Ren, "Constrained consensus in unbalanced networks with communication delays," IEEE Transactions on Automatic Control, vol. 59, no. 3, pp. 775-781, 2014.

[9] A. J. Marasco, S. N. Givigi, and C. A. Rabbath, "Model predictive control for the dynamic encirclement of a target," in Proceedings of the American Control Conference (ACC '12), pp. 2004-2009, Montreal, Canada, June 2012.

[10] F. Chen, W. Ren, and Y. Cao, "Surrounding control in cooperative agent networks," Systems \& Control Letters, vol. 59, no. 11, pp. 704-712, 2010.
[11] T. Wei and X. Chen, "Collective surrounding control in multiagent networks," Chinese Physics B, vol. 23, no. 5, Article ID 050201, 4 pages, 2014.

[12] P. Lin and Y. Jia, "Consensus of second-order discrete-time multi-agent systems with nonuniform time-delays and dynamically changing topologies," Automatica, vol. 45, no. 9, pp. 21542158, 2009.

[13] P. Lin, W. Ren, and Y. Song, "Distributed multi-agent optimization subject to nonidentical constraints and communication delays," Automatica, vol. 65, pp. 120-131, 2016.

[14] P. Lin and Y. Jia, "Multi-agent consensus with diverse timedelays and jointly-connected topologies," Automatica, vol. 47, no. 4, pp. 848-856, 2011.

[15] Y. Hong, L. Gao, D. Cheng, and J. Hu, "Lyapunov-based approach to multiagent systems with switching jointly connected interconnection," IEEE Transactions on Automatic Control, vol. 52, no. 5, pp. 943-948, 2007.

[16] P. Lin, K. Qin, Z. Li, and W. Ren, "Collective rotating motions of second-order multi-agent systems in three-dimensional space," Systems \& Control Letters, vol. 60, no. 6, pp. 365-372, 2011.

[17] L. Mo, Y. Niu, and T. Pan, "Consensus of heterogeneous multiagent systems with switching jointly-connected interconnection," Physica A, vol. 427, pp. 132-140, 2015.

[18] C. Godsil and G. Royle, Algebraic Graph Theory, vol. 207 of Graduate Texts in Mathematics, Springer, New York, NY, USA, 2001.

[19] S. P. Bhat and D. S. Bernstein, "Continuous finite-time stabilization of the translational and rotational double integrators," IEEE Transactions on Automatic Control, vol. 43, no. 5, pp. 678-682, 1998. 


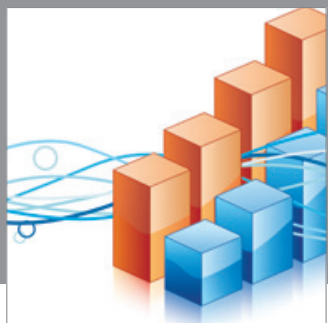

Advances in

Operations Research

mansans

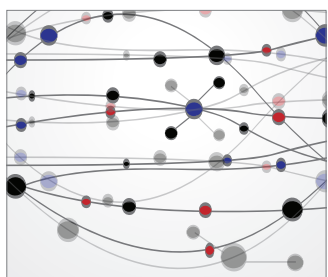

The Scientific World Journal
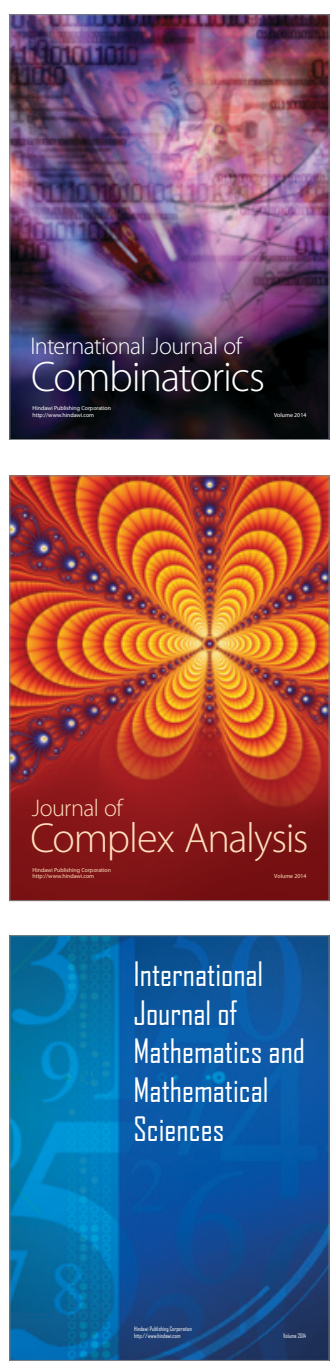
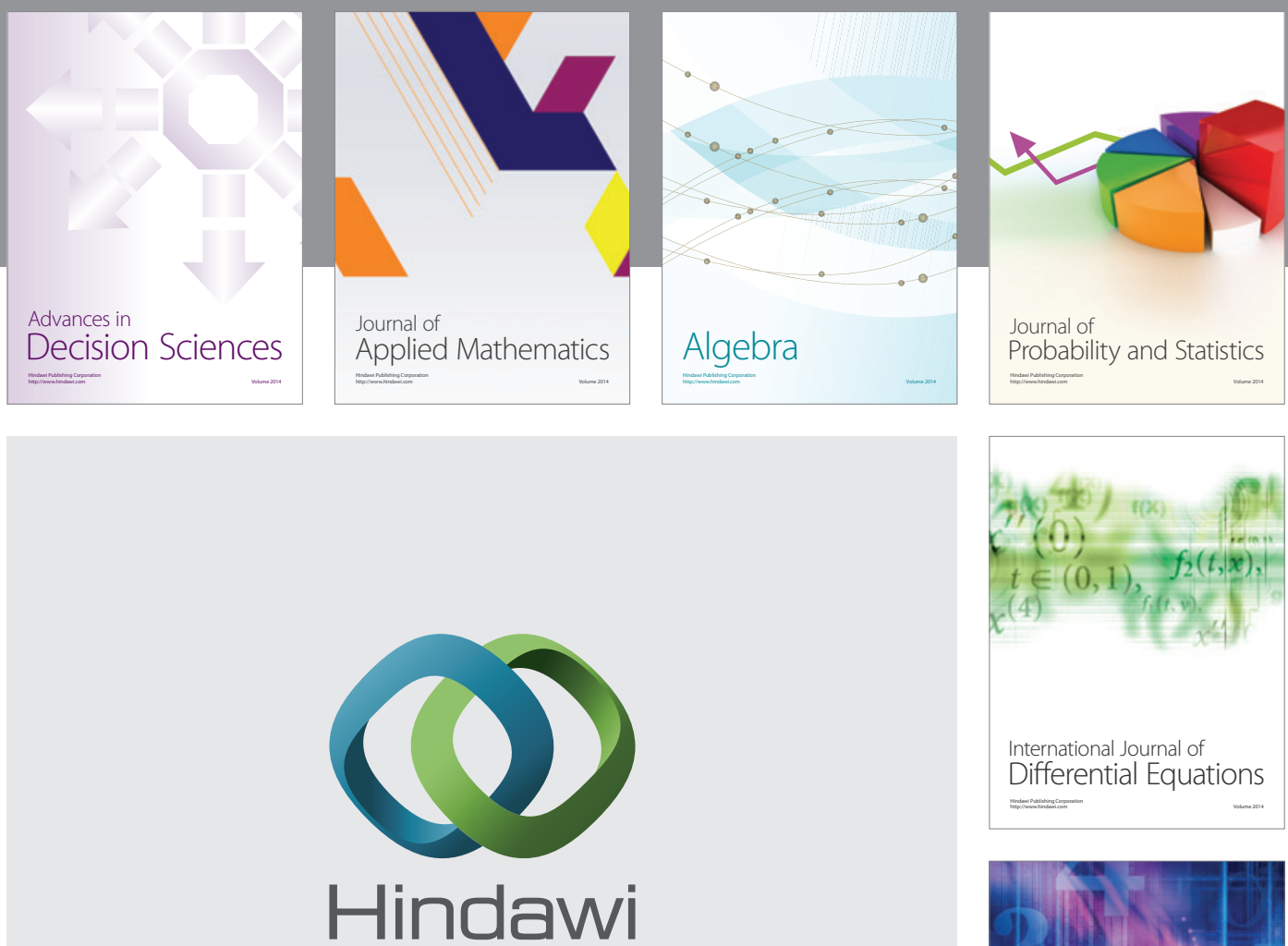

Submit your manuscripts at http://www.hindawi.com
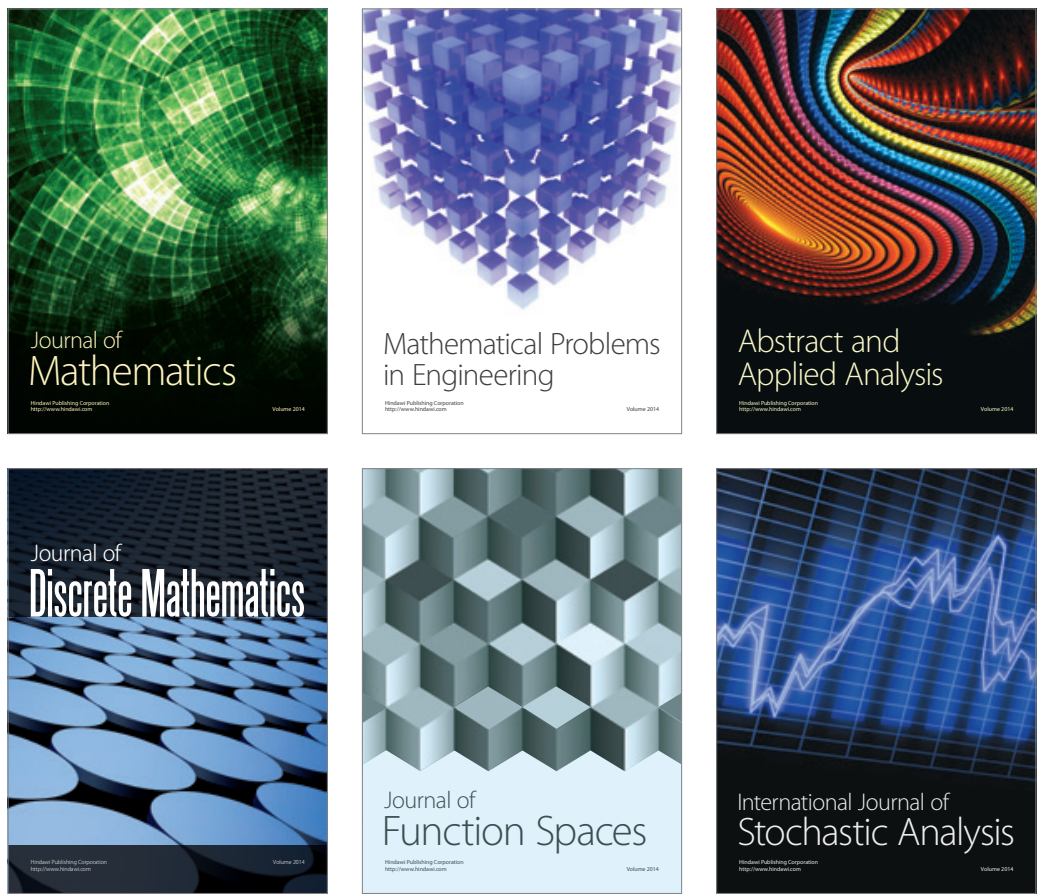

Journal of

Function Spaces

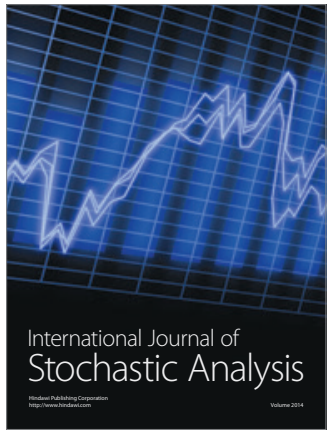

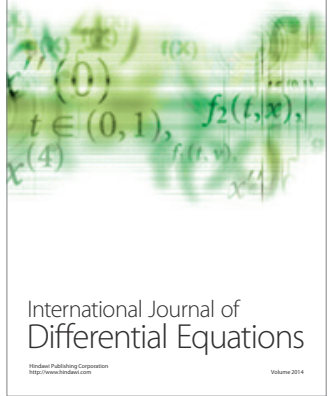
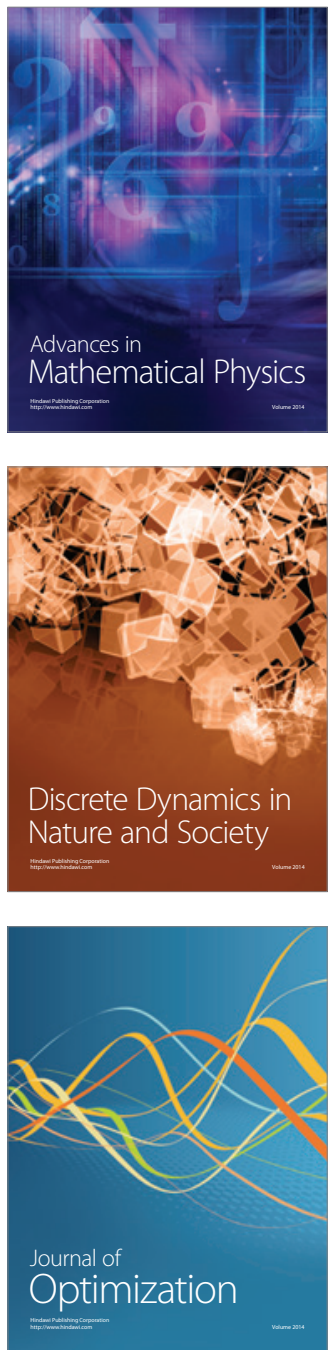